\title{
Posttraumatische Belastungsstörung bei jedem vierten intensivpflichtigen COVID-19-Patienten
}

Um die psychischen Folgen der Pandemie zu untersuchen, haben Forscher über 30.000 Deutsche befragt. Ein weiteres Ergebnis neben der hohen PTBS-Erkrankungsrate: Coronaskeptiker fürchten COVID-19 genauso wie alle anderen.

Coronaskeptiker und -leugner fürchten sich genauso vor COVID-19 wie die Allgemeinbevölkerung, und ein Viertel der schwer Erkrankten leidet im Verlauf unter einer posttraumatischen Belastungsstörung (PTBS). Das sind nur zwei Befunde, die Forscher von der Universität Duisburg-Essen bei einer deutschlandweiten, weitgehend internetbasierten Befragung von über 30.000 Bürgern erhoben haben.

Beim Deutschen Kongress für Psychosomatische Medizin und Psychotherapie berichteten sie jetzt darüber. „Wir haben eine Zeit, die ist ganz anders", sagte Studienleiter Prof. Dr. Martin Teufel, Direktor der Klinik für Psychosomatische Medizin und Psychotherapie, LVR-Klinikum Essen, bei einer Pressekonferenz. Jeder habe in der "somatischen Krisensituation" der Pandemie gemerkt, welche Auswirkungen sie auf die eigene Psyche haben könne.

Die Daten aus der fortlaufenden Studie decken die Zeit von April 2020 bis März 2021 ab. Stresslevel in der Allgemeinbevölkerung seien vom ersten Lockdown an eigentlich durchgängig erhöht gewesen, so Teufel. Bis zu $65 \%$ der Befragten gaben höheren psychischen Distress an, bis zu $45 \%$ höhere generalisierte Angst, $60 \%$ ausgeprägte Corona-Furcht und bis zu $15 \%$ vermehrte Depressivität.

\section{Zunehmender}

\section{Erschöpfungszustand}

Dass Letztere mit dem zweiten Lockdown ab November 2020 sogar noch zunahmen, ist nach Einschätzung des Facharztes für Psychosomatische Medizin und Psychotherapie einem zunehmenden Erschöpfungszustand zuzuschreiben.

Auch international gehe man von einer etwa 30-prozentigen Depressionsrate aus. „Das heißt noch nicht, dass die klinische Diagnose einer Depression erfüllt ist", räumte Teufel ein. „Viele Menschen machen das gut und kommen da auch gut raus."

Psychisch besonders belastet waren Frauen, Jüngere und Menschen mit vorbestehenden psychischen Erkrankungen. „Die Kontrolle war weg“, sagte Teufel. Das spiele bei Angststörungen eine Rolle. Während Furcht (etwa beim Überqueren einer roten Ampel überfahren zu werden), schützend wirke, sei das bei generalisierten Angststörungen nicht mehr der Fall.

DieZahl der Menschen mit schwerer generalisierter Angst habe sich in der Pandemie verdoppelt. Anstatt nur vor der Ansteckung mit dem Virus hätten Menschen „plötzlich ganz viel Angst bekommen, vor ganz vielen Dingen“.

\section{Schreibtherapie gegen PTBS}

Besonderen Schaden nahmen 25\% der schwerkranken, intensivpflichtigen COVID-Patienten: Trotz körperlicher Genesung traten bei ihnen durchschnittlich ab 100 Tagen nach erfolgreicher Behandlung Symptome einer PTBS auf. Das massiv bedrohliche Erlebnis, keine Luft mehr zu bekommen, kommt dabei, ähnlich einem Flashback, als Intrusion zurück: Plötzlich ergreift die Patienten ein Gefühl von Hilflosigkeit, Ausgeliefertsein, Kontrollverlust.

Gegensteuern lässt sich etwa mit einer angeleiteten Schreibtherapie. Patienten arbeiten dabei das Erlebte neu auf, um die Kontrolle über ihre Affekte zurückzuerlangen.

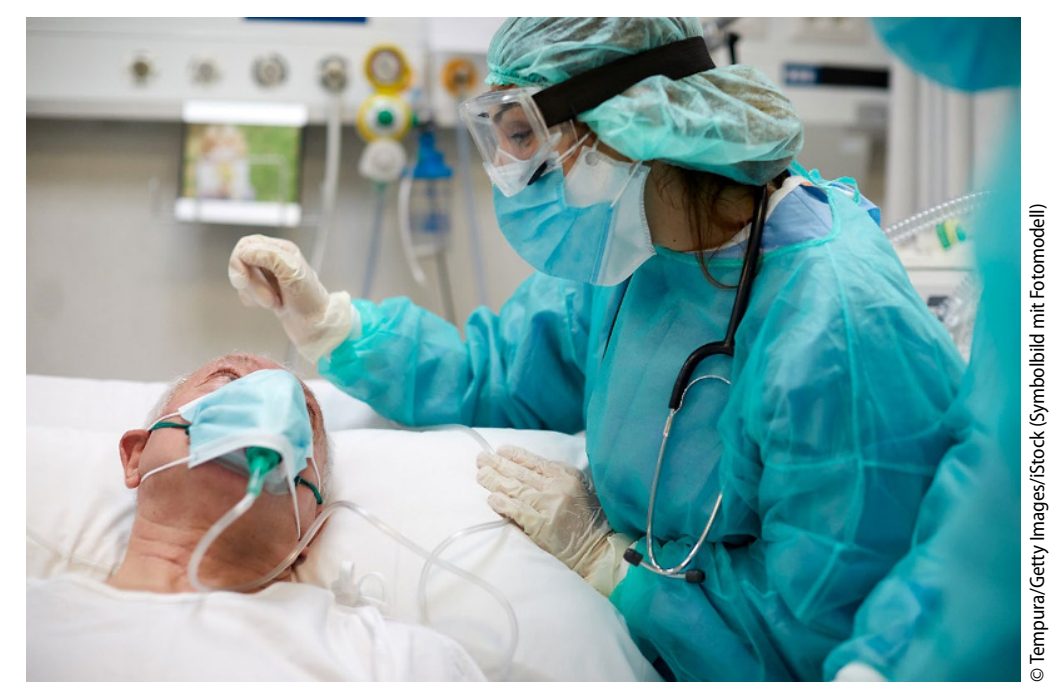

$\Delta$ Das massiv bedrohliche Erlebnis, keine Luft mehr zu bekommen, kann bei intensivpflichtigen COVID-Patienten eine posttraumatische Belastungsstörung auslösen 
Quelle: www. aerztezeitung.de/ Dr. med. Bianca Bach
Unter den Befragten waren auch über 400 Coronaskeptiker. Zum Teil wurden sie von Teufels Team auf Internetforen kontaktiert, wo sie sich über das vermeintlich nicht existierende Virus und Verschwörungstheorien austauschten. Es stellte sich heraus: Sie fürchten sich genauso vor Corona wie die Allgemeinbevölkerung. Sie gaben sogar mehr Depressivität und generalisierte Angst an.

\section{Sorgen ernst nehmen}

Der scheinbare Widerspruch zu ihrer Ablehnung von Schutzmaßnahmen spiegelt einen eigentlich ganz normalen, hier überhandnehmenden Mechanismus der Angstbewältigung: Verdrängung. „Wenn ich sage: ,Das gibt's gar nicht', dann muss ich auch nicht mit der Sorge umgehen."

Statt diese Menschen zu belehren und so in eine Position zu bringen, in der sie das Gefühl haben, sich verteidigen zu müssen, ist es besser, ihre Sorgen ernst zu nehmen und eine evidenzbasierte Auseinandersetzung anzubieten. Information zu suchen und zu erhalten, entpuppte sich übri- gens in der Untersuchung insgesamt als gute Entlastungs-Strategie.

Hinweis des Verlags. Der Verlag bleibt in Hinblick auf geografische Zuordnungen und Gebietsbezeichnungen in veröffentlichten Karten und Institutsadressen neutral.

psychopraxis.neuropraxis2021·24:227-228 https://doi.org/10.1007/s00739-02100744-x

(c) Springer-Verlag GmbH Austria, ein Teil von Springer Nature 2021

\section{S2k-Leitlinie für Diagnostik und Therapie der Multiplen Sklerose}

Am 10. Mai 2021 veröffentlichte die Deutsche Gesellschaft für Neurologie die aktualisierte und erweiterte S2k-Leitlinie zur Diagnostik und Therapie der Multiplen Sklerose (MS). Neu sind unter anderem konkrete Angaben, ob, wann und welche der verlaufsmodifizierenden Immuntherapien indiziert sind. So werden zunehmend neue Substanzen der letzten Jahre Eingang in die tägliche Praxis finden, wodurch die individuell angepasste Behandlung der Multiple Sklerose-Patientinnen und -Patienten weiter verbessert werden kann.

Durch die intensive Multiple Sklerose-Forschung in den letzten Jahren gibt es neue Erkenntnisse zur Diagnostik und zahlreiche neue Therapieoptionen, sodass eine Aktualisierung der Leitlinie von 2012 erforderlich wurde. Herausgeber dieser vollständig überarbeiteten, erweiterten Leitlinie ist die Deutsche Gesellschaft für Neurologie (DGN) [1]. Beteiligte Fachgesellschaften und Organisationen sind die Schweizerische Neurologische Gesellschaft (SNG-SSN), die Österreichische Gesellschaft für Neurologie (ÖGN), der Berufsverband Deutscher Neurologen (BDN), der Berufsverband Deutscher Nervenärzte (BVDN), die Deutsche Fachgesellschaft für Aktivierend-therapeutische Pflege (DGATP), die Deutsche Gesellschaft für Gynäkologie und Geburtshilfe (DGGG), die Deutsche Multiple Sklerose Gesell- schaft (DMSG), der Deutsche Verband für Physiotherapie (ZVK), die Gesellschaft für Neuropädiatrie (GNP), NeurologyFirst, eine unabhängige Initiative von Neurologinnen und Neurologen sowie die Neuromyelitis optica-Studiengruppe (NEMOS). Erstmals haben an der konsensbasierten S2k-Leitlinie auch Betroffene bzw. Patientenvertreterinnen mitgearbeitet. Leitlinienkoordinator und federführender Autor war Prof. Dr. Bernhard Hemmer von der Universitätsklinik der TU München.

\section{Was ist neu?}

Die Multiple Sklerose-Diagnostik wurde durch die Revision der McDonald-Diagnosekriterien 2017 vereinfacht (Liquoruntersuchung sowie MRT-Untersuchung mit definierten Sequenzen; erweiterte Labordiagnostik nur bei entsprechendem kli- nischem Verdacht). Neu wurden der Multiplen Sklerose verwandte Erkrankungen wie die Neuromyelitis-opticaSpektrum-Erkrankungen (NMOSD) und die MOG-IgG-assoziierten Erkrankungen als eigenständige Krankheitsentitäten aufgenommen.

Die verlaufsmodifizierenden MSMedikamente (DMT/,disease modifying therapies") wurden in drei Wirksamkeitskategorien eingeteilt - anstelle des bisherigen Behandlungs-Stufenschemas. Die Eingruppierung erfolgte anhand der Schubratenreduktion aus den Zulassungsstudien. Zur Wirksamkeitskategorie 1 gehören Betainterferone, Dimethylfumarat, Glatirameroide und Teriflunomid, zur Kategorie 2 Cladribin, Fingolimod sowie Ozanimod und zur Kategorie 3 Alemtuzumab, CD20Antikörper (Ocrelizumab, off label Rituximab) und Natalizumab. Mit zuneh- 